Д. С. Сандитов, М. В. Дармаев, В. В. Мантатов. Новый подход к кинетическому критерию стеклования жидкости

УДК 541.64: 539.199

DOI $10.18101 / 2306-2363-2020-1-15-22$

\title{
НОВЫЙ ПОДХОД К КИНЕТИЧЕСКОМУ КРИТЕРИЮ СТЕКЛОВАНИЯ жИДКОСТИ
}

\section{(C) Сандитов Д. С.}

доктор физико-математических наук, профессор,

Бурятский государственный университет имени Доржи Банзарова

Россия, 670000, г. Улан-Удэ, ул. Смолина, 24a

Институт физического материаловедения СО РАН

Россия, 670047, г. Улан-Удэ, ул. Сахьяновой, 6

E-mail: sanditov@bsu.ru

\section{(C) Дармаев М. В.}

кандидат технических наук, доцент,

Бурятский государственный университет имени Доржи Банзарова

Россия, 670000, г. Улан-Удэ, ул. Смолина, 24a

E-mail: darmaev@bsu.ru

\section{(C) Мантатов B. B.}

доктор физико-математических наук, доцент,

Бурятский государственный университет имени Доржи Банзарова

Россия, 670000, г. Улан-Удэ, ул. Смолина, 24a

E-mail: manv999@rambler.ru

С привлечением модели делокализованных атомов предлагается обоснование и обобщение критерия стеклования Шмельцера. В отличие от подходов Бартенева и Волькенштейна-Птицына в обобщенном кинетическом критерии стеклования наряду со временем релаксации и скоростью охлаждения расплава появляются температура стеклования и практически универсальная безразмерная постоянная, которая определяется долей флуктуационного объема, замороженной при температуре стеклования. Развито представление о том, что жидкость переходит в стеклообразное состояние, когда скорость ее охлаждения q достигает определенной доли $\mathrm{C}_{\mathrm{g}}$ от характерной скорости охлаждения $\mathrm{q}_{\mathrm{g}}=\left(\mathrm{T}_{\mathrm{g}} / \tau_{\mathrm{g}}\right)$, тесно связанной со временем релаксации структуры $\tau \mathrm{g}$ при температуре стеклования $\mathrm{T}_{\mathrm{g}}$.

Ключевые слова: стеклование; стеклообразное состояние; делокализация атома; критерий стеклования; модель; релаксационная теория; флуктуационный объем.

Для цитирования: Сандитов Д. С., Дармаев М. B., Мантатов В. В. Новый подход к кинетическому критерию стеклования жидкости // Вестник Бурятского государственного университета. Химия. Физика. 2020. Вып. 1. С. 15-22.

Основные закономерности процесса перехода жидкость - стекло в качественном отношении оказываются одинаковыми для различных аморфных веществ независимо от их природы, что подтверждается наличием в области стеклования универсальных правил и уравнений [1-6].

Однако вместе с тем необходимо отметить, что для разных классов стекол при температуре стеклования $T_{g}$ замораживается подвижность различных кинетических единиц, связанных с различным характером взаимодействия между части- 
цами. Стеклование линейных аморфных полимеров является процессом физической релаксации, связанным с потерей сегментальной подвижности при $T_{g}$, а стеклование неорганических стекол с сеточной структурой относится к процессам химической релаксации и обусловлено замораживанием процесса переключения валентных химических связей. Стеклование густосшитых сетчатых полимеров (например, эпоксидов) также имеет свою специфику, хотя в качественном отношении они подчиняются общим универсальным закономерностям [6].

Факт существования универсальных закономерностей означает, что процессы стеклования различных жидкостей могут быть описаны общей теорией, способной предсказывать, например, характер эволюции их макроскопических свойств. Создание такой общей физической теории стеклования представляет собой сложнейшую проблему статистической физики и физики конденсированного состояния (обзоры $[1,2])$. Эта проблема едва ли будет решена в ближайшие десятилетия. Дискуссия о природе стеклования имеет столетнюю историю, она продолжается, начиная с первой половины XX века.

Настоящая работа посвящена анализу и обобщению кинетических критериев стеклования с привлечением модели делокализованных атомов [7-9]. Особое внимание уделено обоснованию критерия Шмельцера $[1,10,11]$.

\section{Кинетические критерии стеклования}

В области стеклования жидкости молекулярные перегруппировки становятся настолько медленными, что изменение структуры расплава при охлаждении не успевает следовать за изменением температуры. При этом довольно резко возрастает время релаксации структуры. Температура стеклования жидкости $T_{g}$ зависит от скорости охлаждения $q=(d T / d t)$. Чем меньше скорость охлаждения, тем при более низкой температуре стеклуется жидкость и, наоборот. Из этих и других данных следует, что стеклование представляет собой ярко выраженный релаксационный процесс и подчиняется кинетическим закономерностям.

С точки зрения релаксационного подхода в процессе стеклования жидкости решающую роль играет соотношение между временем релаксации структуры $\tau$ и скоростью охлаждения расплава $q$ [1-6]. В 1951 г. Бартенев [12], исходя из общих соображений, предложил следующую связь между этими величинами

$$
q \tau_{g}=C
$$

где $\tau_{g}-$ время релаксации при температуре стеклования $T_{g}, C-$ эмпирический параметр. Здесь и далее под $q$ следует понимать абсолютную величину скорости изменения температуры $|q|$ при охлаждении расплава или нагревании стекла: $|d T / d t|$.

Волькенштейн и Птицын [13] разработали физическую теорию, в соответствии с которой частицы системы могут находиться в двух состояниях, разделенных энергетическим барьером. Энергия возбужденного состояния 2 с долей частиц $n_{2}$ больше энергии основного состояния 1 с долей частиц $n_{1}$ на величину $\Delta E=U_{1}-U_{2}$, где $U_{1}, U_{2}-$ кинетические барьеры для переходов $1 \rightarrow 2$ и $2 \rightarrow 1$, соответственно. Решение составленного кинетического уравнения приводит к заключению, что при некоторой температуре $T_{g}$ происходит замораживание значения $n_{2}-$ стеклование системы. 
Д. С. Сандитов, М. В. Дармаев, В. В. Мантатов. Новый подход к кинетическому критерию стеклования жидкости

Температура стеклования $T_{g}$ отвечает условию [13]

$$
\left.\frac{d \tau}{d T}\right|_{T=T_{g}}=-\frac{1}{q} .
$$

В теории Волькенштейна-Птицына критерием перехода жидкость-стекло служит уравнение стеклования

$$
q \tau_{g}=\delta T_{g} .
$$

Обозначение правой части этого равенства $\delta T_{g}$ предложено Немиловым [14]. Величина $\delta T_{g}$ трактуется как полоса температур, характеризующая интервал перехода от жидкости к стеклу при охлаждении. Параметр уравнения Бартенева $C$ приобретает смысл полосы температур $\delta T_{g}$.

Шмельцер с соавторами $[1,10,11]$ вводят характерное время изменения температуры

$$
\tau_{T}=\frac{T}{q}=T\left(\frac{d T}{d t}\right)^{-1}
$$

и рассматривают соотношение между этим характерным временем $\tau_{T}$ и временем релаксации структуры $\tau$. При $\tau<<\tau_{T}$ система находится в состоянии термодинамического равновесия, а при $\tau>>\tau_{T}$ состояние системы заморожено - стеклование завершено. По предположению Шмельцера жидкость переходит в стеклообразное состояние, когда время структурной релаксации $\tau$ становится равным характерному времени изменения температуры $\tau_{T}$.

Таким образом, исходя из этих общих соображений, Шмельцером сформулирован следующий кинетический критерий стеклования $[1,10,11]$

$$
\tau \approx \tau_{T}, \quad\left[\frac{1}{T}\left(\frac{d T}{d t}\right) \tau\right]_{T=T_{g}}=C_{3}, \quad C_{3} \approx 1 .
$$

\section{Обоснование и обобщение критерия стеклования Шмельцера в рамках модели делокализованных атомов}

Температурная зависимость времени релаксации в области стеклования успешно описывается уравнением Вильямса-Ландела-Ферри (ВЛФ) [15]

$$
\ln \frac{\tau(T)}{\tau\left(T_{g}\right)}=-C_{1} \frac{T-T_{g}}{T-T_{g}+C_{2}} .
$$

Подстановка $\tau(T)$ из этого уравнения в условие перехода жидкость-стекло (2) приводит к равенству [16]

$$
q \tau_{g}=\frac{C_{2}}{C_{1}}
$$

где параметры уравнения ВЛФ $C_{1}$ и $C_{2}$ имеют следующий физический смысл [7]

$$
C_{1}=\frac{1}{f_{g}}, \quad C_{2}=\frac{f_{g}}{\beta_{f}},
$$


причем произведение $T_{g}$ и коэффициента теплового расширения флуктуационного объема при температуре стеклования $\beta_{f}$ является однозначной функцией доли флуктуационного объема $f_{g}$, замороженной при температуре стеклования [7],

$$
\beta_{f} T_{g}=f_{g} \ln \left(1 / f_{g}\right) \text {. }
$$

Флуктуационный объем аморфного вещества $\Delta V_{e}$ обусловлен тепловыми смещениями атомов [7]

$$
\Delta \mathrm{V}_{\mathrm{e}}=\mathrm{N}_{\mathrm{e}} \Delta \mathrm{V}_{\mathrm{e}},
$$

где $N_{e}$ - число делокализованных атомов (кинетических единиц), $\Delta \mathrm{v}_{e}-$ элементарный флуктуационный объем, необходимый для делокализации атома - его предельного смещения из локального равновесного положения. Объемная доля флуктуационного объема $f_{g}$, замороженная при температуре стеклования $T_{g}$, слабо зависит от природы аморфных веществ [7, 9] (табл. 1)

$$
f_{g}=\left(\frac{\Delta V_{e}}{V}\right)_{T=T_{g}} \approx 0.020-0.030
$$

У стекол одного класса величина $f_{g}$ оказывается практически универсальной постоянной $f_{g} \approx$ const.

Из соотношений (5)-(7) получаем следующую интерпретацию уравнения стеклования (3)

Таблица

\begin{tabular}{|c|c|c|c|c|c|c|}
\hline Аморфное вещество & $\begin{array}{c}T_{g}, \\
\mathrm{~K}\end{array}$ & $\mathrm{C}_{1}$ & $\begin{array}{c}C_{2}, \\
\mathrm{~K}\end{array}$ & $\begin{array}{c}\delta T_{g}=\frac{C_{2}}{C_{1}}, \\
\mathrm{~K}\end{array}$ & $f_{g}=\frac{1}{C_{1}}$ & $\begin{array}{c}C_{g} \cdot 10^{3}, \\
\mathrm{~K}\end{array}$ \\
\hline \multicolumn{7}{|c|}{ Натриевосиликатные стекла $\mathrm{Na}_{2} \mathrm{O}-\mathrm{SiO}_{2}$} \\
\hline $\mathrm{Na}_{2} \mathrm{O}$, мол. \% 15 & 782 & 36 & 430 & 12 & 0.028 & 7.8 \\
\hline 20 & 759 & 36 & 390 & 11 & 0.028 & 7.8 \\
\hline 25 & 739 & 35 & 355 & 10 & 0.028 & 7.8 \\
\hline 30 & 721 & 35 & 322 & 9 & 0.028 & 7.8 \\
\hline 33 & 712 & 35 & 304 & 9 & 0.028 & 7.8 \\
\hline 35 & 705 & 35 & 291 & 8 & 0.028 & 7.8 \\
\hline \multicolumn{7}{|c|}{ Аморфные полимеры } \\
\hline Полиизобутилен & 202 & 38 & 104 & 2.7 & 0.026 & 7.1 \\
\hline Поливинилацетат & 305 & 36 & 47 & 1.3 & 0.028 & 7.8 \\
\hline Поливинилхлорацетат & 296 & 40 & 40 & 1.0 & 0.025 & 6.8 \\
\hline Полиметилакрилат & 276 & 42 & 45 & 1.1 & 0.024 & 6.4 \\
\hline Полиуретан & 238 & 36 & 33 & 0.9 & 0.028 & 7.8 \\
\hline Натуральный каучук & 300 & 38 & 54 & 1.4 & 0.026 & 7.1 \\
\hline $\begin{array}{l}\text { Метакрилатные полимеры } \\
\text { этиловый }\end{array}$ & 335 & 40 & 65 & 16 & 0.025 & 68 \\
\hline н-бутиловый & 300 & 39 & 97 & 2.5 & 0.026 & 7.1 \\
\hline Н-октиловый & 253 & 37 & 107 & 2.9 & 0.027 & 7.5 \\
\hline
\end{tabular}

Параметры уравнения Вильямса-Ландела-Ферри $C_{1}, C_{2}$ и характеристики стеклования аморфных веществ [9] 
Д. С. Сандитов, М. В. Дармаев, В. В. Мантатов. Новый подход к кинетическому критерию стеклования жидкости

\begin{tabular}{|l|c|c|c|c|c|r|}
\hline \multicolumn{7}{|c|}{ Металлические стекла } \\
\hline $\mathrm{Pd}_{40} \mathrm{Ni}_{40} \mathrm{P}_{20}$ & 602 & 39 & 93 & 2.4 & 0.026 & 7.1 \\
$\mathrm{Pt}_{60} \mathrm{Ni}_{15} \mathrm{P}_{25}$ & 500 & 37 & 95 & 2.6 & 0.027 & 7.5 \\
$\mathrm{Pd}_{77.5} \mathrm{Cu}_{6} \mathrm{Si}_{16.5}$ & 653 & 38 & 100 & 2.6 & 0.026 & 7.1 \\
$\mathrm{Fe}_{80} \mathrm{P}_{13} \mathrm{C}_{7}$ & 736 & 38 & 120 & 3.2 & 0.026 & 7.1 \\
\hline \multicolumn{7}{|c|}{ Низкомолекулярные органические стекла } \\
\hline Пропанол & 98 & 41 & 25 & 0.6 & 0.024 & 6.4 \\
Пропиленгликоль & 160 & 44 & 40 & 0.9 & 0.023 & 6.1 \\
Глицерин & 185 & 42 & 53 & 1.3 & 0.024 & 6.4 \\
Канифоль & 303 & 36 & 29 & 0.8 & 0.028 & 7.8 \\
Изобутиловый спирт & 118 & 38 & 38 & 1.0 & 0.026 & 7.1 \\
\hline
\end{tabular}

$$
q \tau_{g}=\frac{f_{g}}{\ln \left(1 / f_{g}\right)} T_{g}
$$

Разделив обе части этого равенства на температуру стеклования $T_{g}$, приходим к обобщенному кинетическому критерию стеклования

$$
\frac{q \tau_{g}}{T_{g}}=\frac{f_{g}}{\ln \left(1 / f_{g}\right)}=C_{g} \cong \text { const } \approx 7 \cdot 10^{-3}
$$

который можно записать в виде выражения для определения температуры перехода $T_{g}$

$$
\left.\left(\frac{1}{T}\left|\frac{d T}{d t}\right| \tau\right)\right|_{T=T_{g}}=C_{g}, \quad C_{g} \approx 7 \cdot 10^{-3} .
$$

Данное соотношение можно рассматривать как обоснование и модификацию критерия стеклования Шмельцера (4). При этом постоянная $C_{3}$ приобретает определенный физический смысл

$$
C_{3}=C_{g}=\frac{f_{g}}{\ln \left(1 / f_{g}\right)} \approx \text { const } \approx 7 \cdot 10^{-3}
$$

Тем самым устраняется недостаток критерия (4), сводящийся к приравниванию постоянной $C_{3}$ единице $C_{3} \approx 1$, причем фактически в виде постулата без особого обоснования. Легко видеть, что критерий Шмельцера (4) приводит к ошибочному равенству

$$
q \tau_{g} \approx T_{g},
$$

которое противоречит уравнению стеклования (3), следующему из теории Волькенштейна-Птицына. Например, у силикатных стекол при $T_{g} \approx 800 \mathrm{~K}$ произведение $q \tau_{g}$, согласно данному критерию (4), должно соответствовать величине: $q \tau_{g} \approx$ $800 \mathrm{~K}$ [14], тогда как типичные значения произведения $q \tau_{g}$ У этих стекол составляют около [9]: $\mathrm{q} \tau_{\mathrm{g}} \approx(5 \div 10) \mathrm{K}$.

Обобщенный кинетический критерий стеклования (10) для $q \tau_{g}$ дает значения

$$
q \tau_{g}=C_{g} T_{g} \approx 5-6 \mathrm{~K},
$$

которые находятся в согласии с типичными данными для произведения $q \tau_{g}$. 


\section{Формулировка обобщенного кинетического критерия стеклования}

В отличие от подходов Бартенева (1) и Волькенштейна-Птицына (3) в обобщенном кинетическом критерии стеклования (9) наряду со скоростью охлаждения $q$ и временем релаксации $\tau_{g}$ появляется температура перехода $T_{g}$.

Предлагаемый нами обобщенный критерий перехода жидкость-стекло (9) можно сформулировать следующим образом: жидкость переходит в замороженное стеклообразное состояние, когда скорость ее охлаждения $q$ достигает определенной постоянной доли $C_{g}$ от характерной скорости охлаждения $q_{g}=\left(T_{g} / \tau_{g}\right)$, тесно связанной со временем релаксации структуры $\tau_{g}$ при температуре стеклования $T_{g}$,

$$
\frac{q}{q_{g}}=\frac{q}{\left(T_{g} / \tau_{g}\right)}=C_{g} \approx \text { const } \approx 7 \cdot 10^{-3} .
$$

При этом время релаксации структуры $\tau_{g}$ достигает доли $C_{g}$ от характерного времени изменения температуры $\tau_{\mathrm{T}}=\left(\mathrm{T}_{\mathrm{g}} / \mathrm{q}\right)$ :

$$
\frac{\tau_{g}}{\tau_{T}}=\frac{\tau_{g}}{\left(T_{g} / q\right)}=C_{g} \approx \text { const } \approx 7 \cdot 10^{-3}
$$

\section{Заключение}

Предложено обобщение кинетических критериев стеклования Бартенева (1), Волькенштейна-Птицына (3), Шмельцера (4). Показано, что эмпирическая постоянная $C_{3}$ в критерии Шмельцера (4) определяется долей флуктуационного объема $f_{g}$, замороженной при температуре стеклования $T_{g}$, и составляет около $C_{3}$ $\approx$ const $\approx 7 \cdot 10^{-3}$ (вместо $C_{3} \approx 1$ ). Этим самым снимается противоречие между критерием Шмельцера и классической релаксационной теорией стеклования Волькенштейна-Птицына.

Обобщенный кинетический критерий стеклования сводится к утверждению: жидкость переходит в стеклообразное состояние, когда скорость ее охлаждения $q$ достигает доли $C_{g}$ от характерной скорости охлаждения $q_{g}=\left(T_{g} / \tau_{g}\right)$, тесно связанной со временем релаксации структуры $\tau_{g}$ при температуре стеклования $T_{g}$.

Возможна аналогичная, но несколько другая формулировка: жидкость стеклуется при достижении времени структурной релаксации $\tau_{g}$ до постоянной доли $C_{g}$ от характерного времени изменения температуры $\tau_{T}=\left(T_{g} / q\right)$.

Работа выполнена при финансовой поддержке Министерства науки и высшего образования РФ (грант №3.5406.2017/БЧ).

\section{Литература}

1. Тропин Т. В., Шмельцер Ю. В. П., Аксенов В. Л. Современные аспекты кинетической теории стеклования // Успехи физических наук. 2016. Т. 186, № 1. С. 46-73.

2. Сандитов Д. С., Ожован М. И. Релаксационные аспекты перехода жидкость стекло // Успехи физических наук. 2019. Т. 189, № 2. С. 113-133.

3. Сандитов Д. С., Бартенев Г. М. Физические свойства неупорядоченных структур. Новосибирск: Наука. 1982. 259 с. 
Д. С. Сандитов, М. В. Дармаев, В. В. Мантатов. Новый подход к кинетическому критерию стеклования жидкости

4. Ростиашвили В. Г., Иржак В. И., Розенберг Б. А. Стеклование полимеров. Л.: Химия, 1987. $192 \mathrm{c.}$

5. Мазурин О. В. Стеклование. Л.: Наука, 1986. 158 с.

6. Бартенев Г. М., Бартенева А. Г. Релаксационные свойства полимеров. М.: Химия, 1992. $382 \mathrm{c}$.

7. Сандитов Д. С. Модель делокализованных атомов в физике стеклообразного состояния // ЖЭТФ. 2012. Т. 142, Вып. 1(7). С. 123-137.

8. Сандитов Д. С., Дармаев М. В., Сандитов Б. Д. О релаксационной природе стеклования аморфных полимеров и низкомолекулярных аморфных веществ // Физика твердого тела. 2015. Т. 57, Вып. 8. С. 1629-1634.

9. Сандитов Д. С., Дармаев М. В., Сандитов Б. Д. Температурный интервал переходи жидкости в стекло // Физика твердого тела. 2016. Т. 58, Вып. 2. С. 372-376.

10. Schmelzer J. W. P. Kinetic criteria of glass formation and pressure dependence of the glass transition temperature // J. Chem. Phys. 2012. V. 136. P. 074512.

11. Schmelzer J. W. P. Kinetic criteria of glass formation, pressure dependence of the glass transition temperature, and the Prigogine - Defay ratio // J. Non-Crystall. Solids. 2015. V. 407. P. 170-178.

12. Бартенев Г. М. О зависимости между температурой стеклования силикатного стекла и скоростью охлаждения или нагревания // ДАН СССР. 1951. Т. 76, № 2. С. 227230.

13. Волькенштейн М. В., Птицын О. Б. Релаксационная теория стеклования // Ж. технической физики. 1956. Т. 26, № 10. С. 2204-2222.

14. Немилов С. В. Уравнение Максвелла и классические теории стеклования как основа прямого расчета вязкости при температуре стеклования // Физика и химия стекла. 2013. T. 39, № 6. С. 857-878.

15. Ферри Дж. Вязкоупругие свойства полимеров. М.: ИЛ, 1963. 535 с.

\section{A NEW APPROACH TO THE KINETIC CRITERION OF GLASS TRANSITION OF LIQUID}

Sanditov D. S.

Doctor of Physical and Mathematical Sciences, Professor

Buryat State University

670000, Ulan-Ude, Smolina, Str., 24a

Institute of Physical Materials Science SB RAS

670047, Ulan-Ude, Sakhyanovoi, Str., 6

E-mail: sanditov@bsu.ru

Darmaev M. V.

Candidate of Technical Sciences

Buryat State University

670000, Ulan-Ude, Smolina, Str., 24a.

E-mail: darmaev@bsu.ru

Mantatov $V . V$.

Doctor of Physical and Mathematical Sciences, associate Professor

Buryat State University

670000, Ulan-Ude, Smolina, Str., 24a.

E-mail:manv999@rambler.ru 
Using the model of delocalized atoms, we propose justification and generalization of the Schmelzer glass transition criterion. In contrast to the Bartenev and Wolkenstein-Ptitsyn approaches, the generalized kinetic criterion of glass transition, along with the relaxation time and the melt cooling rate, shows a glass transition temperature and an almost universal dimensionless constant, which is determined by the fraction of the fluctuating volume frozen at the glass transition temperature. The idea is developed that a liquid passes into a glassy state when its cooling rate $\mathrm{q}$ reaches a certain proportion of $\mathrm{C}_{\mathrm{g}}$ from the characteristic cooling rate $\mathrm{q}_{\mathrm{g}}=\left(\mathrm{T}_{\mathrm{g}} / \mathrm{T}_{\mathrm{g}}\right)$, which is closely related to the relaxation time of the structure $\mathrm{T}_{\mathrm{g}}$ at the glass transition temperature $\mathrm{T}_{\mathrm{g}}$.

Keywords: glass transition, glassy state; atom delocalization; glass transition criterion; model; relaxation theory; fluctuation volume. 